\title{
Perception of socket alignment perturbations in amputees with transtibial prostheses
}

\author{
David A. Boone, PhD; ${ }^{1-2 *}$ Toshiki Kobayashi, PhD; ${ }^{1-2}$ Teri G. Chou, PhD; ${ }^{1}$ Adam K. Arabian, PhD; ${ }^{1}$ Kim L. \\ Coleman, MS; ${ }^{1}$ Michael S. Orendurff, PhD; ${ }^{1}$ Ming Zhang, $\mathbf{P h D}^{\mathbf{2}}$ \\ ${ }^{1}$ Orthocare Innovations, Mountlake Terrace, WA; ${ }^{2}$ Department of Health Technology and Informatics, The Hong Kong \\ Polytechnic University, Hong Kong, People's Republic of China
}

\begin{abstract}
A person with amputation's subjective perception is the only tool available to describe fit and comfort to a prosthetist. However, few studies have investigated the effect of alignment on this perception. The aim of this article is to determine whether people with amputation could perceive the alignment perturbations of their prostheses and effectively communicate them. A randomized controlled perturbation of angular (3 and 6 degrees) and translational (5 and $10 \mathrm{~mm}$ ) alignments in the sagittal (flexion, extension, and anterior and posterior translations) and coronal (abduction, adduction, and medial and lateral translations) planes were induced from an aligned condition in 11 subjects with transtibial prostheses. The perception was evaluated when standing (static) and immediately after walking (dynamic) using software that used a visual analog scale under each alignment condition. In the coronal plane, Friedman test demonstrated general statistical differences in static $(p<0.001)$ and dynamic $(p<0.001)$ measures of perceptions with angular perturbations. In the sagittal plane, it also demonstrated general statistical differences in late-stance dynamic measures of perceptions $(p<0.001)$ with angular perturbations, as well as in early-stance dynamic measures of perceptions $(p<0.05)$ with translational perturbations. Fisher exact test suggested that people with amputation's perceptions were good indicators for coronal angle malalignments but less reliable when defining other alignment conditions.
\end{abstract}

Key words: alignment, amputation, below-knee prosthesis, instrumentation, malalignment, perception, pressure, socket, transtibial prosthesis, visual analog scale.

\section{INTRODUCTION}

Alignment of the socket and shank is important for the optimal prosthetic function and comfort of a person with amputation by influencing the weight-bearing load between the residual limb and the ground. In general, the literature supports the idea that successful alignment plays one of the key roles in successful prosthetic fit [1-5]. To achieve the best prosthetic function for each person with amputation, the prosthetist is trained to align the prosthesis to counteract the tendency of the socket to rotate about the residual limb and maximize comfort while walking [6]. For instance, the prosthetic socket may be set up slightly laterally from the neutral position in the coronal plane to generate a slight genu varum moment in stance to relieve pressure over the head of the fibula and peroneal nerve and to keep trunk stability by reducing lateral bending over the foot [7-8]. Thus, the experience of prosthetic function is in part affected by alignment and not just by its geometry, material, or structure.

\footnotetext{
Abbreviations: PAPI $=$ Prosthesis Alignment Perception Instrument, VAS = visual analog scale.

*Address all correspondence to David A. Boone, PhD; Orthocare Innovations, 6405 218th St SW, Suite 301, Mountlake Terrace, WA 98043; 800-672-1710; fax: 206219-1144. Email: dboone@orthocareinnovations.com http://dx.doi.org/10.1682/JRRD.2011.08.0143
} 
Perception of the person with amputation regarding the prosthetic fit and function is also an important aspect of determining the best alignment. Therefore, prosthetists usually take verbal feedback during the dynamic alignment process to assess the person with amputation's satisfaction. Some individuals are better at communicating their perceived issue with the prosthesis with regard to comfort and performance; some cannot provide very specific information to aid the prosthetist in achieving a fully optimized prosthetic limb system. Prosthetists generally evaluate sensations of static and dynamic balance in the sagittal and coronal planes, but there is no definitive or quantifiable method to obtain this essential information.

Previous studies used perception to evaluate prosthetic functions to evaluate the effect of a teletorsion device [9], pylon flexibility [10], prosthetic knee [1112], weight of modular prosthetic components, [13] and prosthetic feet [14-16]. One of the validated ways that has been used in a wide range of studies to evaluate a person with amputation's perception of lower-limb prosthetic function has been the Prosthesis Evaluation Questionnaire [17]. Interestingly, Hafner et al. claimed in their review article on prosthetic feet that the subject perception measures appeared to be valid to clinically support the use of a certain type of prosthetic foot over another [18].

Minimal research has been reported regarding the effect of the alignment on a person with amputation's perception of fit and comfort. Hobson reported the effect of a powered alignment device that allowed the participants with amputation ( $n=12$ ) to adjust the alignment of their foot [19]. Some of the interesting findings of his study included-

1. All persons with amputation could arrive at their preferred alignment.

2. Disagreement often existed between the prosthetist and the person with amputation on a preferred alignment.

3. Socket fit had a significant effect on a person with amputation's sensitivity to alignment.

4. Only a few steps were required for persons with amputation to detect malalignments.

5. Persons with amputation were unable to consistently align a prosthetic foot within $1^{\circ}$ of angulation.

6. Best alignment was achieved through collaboration between the prosthetist and the person with amputation. However, Zahedi et al. demonstrated that both prosthetists and persons with amputation would accept a range of alignment as optimal [2].
To increase our understanding of transtibial prosthetic malalignment, it would be important to measure the persons with amputation's feedback regarding the perception of the alignment. The fundamental premise to quantify their perceptions was that they could directly feel and respond to the mechanical effects caused by alignment perturbations. This would be supported by the reports of changes in socket pressure with alignment perturbations [20-21]. The aim of this article was therefore to investigate whether the individuals with transtibial prostheses could perceive perturbation in the alignment of their prostheses and communicate it effectively. The hypothesis of this article was that the individuals with transtibial prostheses could perceive and communicate the nature of socket alignment in the sagittal and coronal planes.

\section{METHODS}

\section{Subjects}

Eleven individuals with unilateral transtibial prostheses participated in this study (10 male and 1 female; age [mean \pm standard deviation]: $47 \pm 13$ yr; height: $1.68 \pm$ $0.08 \mathrm{~m}$; weight: $70 \pm 16 \mathrm{~kg}$; residual-limb length: $0.13 \pm$ $0.01 \mathrm{~m}$ ). Six subjects had an amputation on the left leg, and five subjects had an amputation on the right leg. Inclusion criteria were (1) use of a modular endoskeletal prosthesis for more than 1 year, (2) bilateral normal range of motion of the lower limb, (3) ability to walk for the period of the alignment perturbation trials, (4) ability to walk without additional assistive devices, (5) ability to communicate individual perceptions, and (6) ability to provide informed consent. The cause of amputation was trauma in 10 subjects and peripheral vascular disease in 1 subject. Surgical techniques for amputation varied among the subjects. A SACH (solid ankle cushion heel) foot was used in all subjects.

\section{Instrumentation}

We used the Prosthesis Alignment Perception Instrument (PAPI) to measure subjects' perception of alignment. The PAPI consisted of both an alignment device and software.

\section{Alignment Device}

The alignment device allowed the prosthetist to perform independent tunings of translation and angulation in 
the sagittal and coronal planes, rotation in the transverse plane, and adjustment of the height. The prosthetist used two tilt sensors (CXT-02 biaxial tilt sensor, Moog Crossbow; Milpitas, California) to compute the relative offsets of the angles between the socket and shank. The prosthetist could use this information to consistently achieve and record the alignment conditions described in the "Procedure" section. The translation was adjusted using a sagittal and coronal translation adjuster with millimeter scale (model 10A40/A, Streifeneder ortho.production GmbH; Emmering, Germany). The details of the alignment device are described elsewhere [22].

\section{Software}

The PAPI's software interface quantified and digitized the subject's response to typical questions on how the alignment felt using an interactive visual analog scale (VAS) (Figure 1). It was designed to measure the subject's perception consistently and easily under different alignment conditions. The software interface consists of a VAS and pop-up menu choices in order to transform the relative location of the VAS marker to a scale of -100 to +100 . The end points of the VAS were indicated by a text description of an extreme sensation that is suitable for each prosthetic malalignment (Figure 1, Table 1). The face validity of the VAS used is that the questions are

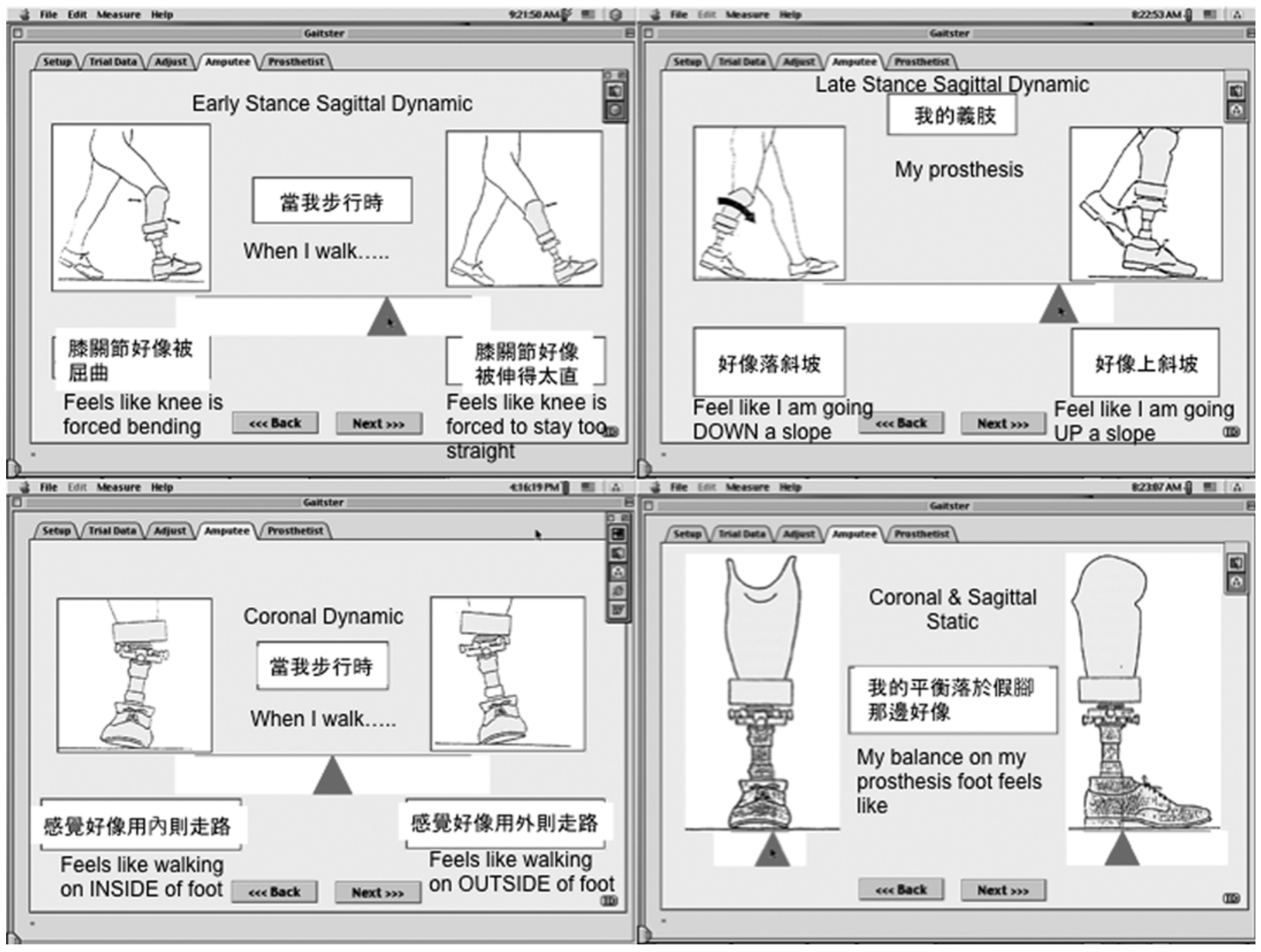

Figure 1.

Prosthesis Alignment Perception Instrument software interface of questions intended to indentify specific prosthetic malalignment. 
JRRD, Volume 49, Number 6, 2012

Table 1.

Prosthesis Alignment Perception Instrument (PAPI) question descriptions intended for specific prosthetic malalignment.

\begin{tabular}{llc}
\hline \multicolumn{1}{c}{ PAPI Variable } & \multicolumn{1}{c}{ Description } & Intended Alignment Change to be Measured \\
\hline Coronal Static & Mediolateral balance (standing) & Coronal angle, coronal translation \\
Coronal Dynamic & Socket abduction/adduction (midstance) & Coronal angle, coronal translation \\
Sagittal Static & Anteroposterior standing balance & Sagittal angle, sagittal translation \\
Early-Stance Sagittal Dynamic & Heel moment (early stance) & Sagittal angle, sagittal translation \\
Late-Stance Sagittal Dynamic & Toe moment (terminal stance) & Sagittal angle, sagittal translation \\
\hline \hline
\end{tabular}

what are usually asked of a person with amputation by a prosthetist during the alignment tuning procedure. We selected each wording for clarity based on subjects' perception and level of education, and three prosthetists reviewed it during development of the PAPI.

The prosthetist presented questions using both text and graphics after each alignment trial. The subjects provided answers to each question by adjusting a sliding marker on a computer-generated VAS. Each of the screens shown in Figure 1 were presented following each alignment change. Three screens (early-stance sagittal dynamic, late-stance sagittal dynamic, and coronal dynamic) were shown following the dynamic condition (walking portion of the study), while one screen (coronal and sagittal static) was shown following the static condition (standing portion of the study). Table 1 lists the VAS question descriptions prepared for each alignment perturbation tested. Subjects were able to move forward or backward throughout the questions using navigation buttons until they reached satisfaction. Because the majority of the subjects who participated in this study were not able to speak English, the software was designed to display the questions in either English or Chinese.

\section{Procedure}

Both the prosthetist and subject aligned the prosthesis to their satisfaction. A single prosthetist aligned the prosthesis for all subjects. We considered this alignment to be the nominally aligned condition. From there, we performed randomized controlled perturbations of angular $\left(3^{\circ}\right.$ and $\left.6^{\circ}\right)$ and translational (5 and $10 \mathrm{~mm}$ ) alignment in the sagittal (flexion, extension, and posterior and anterior translation) or coronal (adduction, abduction, and medial and lateral translation) planes [22]. Therefore, we tested a total of 17 alignment conditions (16 malaligned and 1 nominally aligned). The subjects were blinded to the alignment perturbations.

We used the VAS in each question to evaluate the subjects' perceptions of various alignment conditions in different domains. The questions accounted for static sensations of standing balance anteroposteriorly and mediolaterally as well as dynamic sensations in early and late stance of gait in the sagittal plane and at midstance in the coronal plane (Table 1). We instructed the subjects to use a computer mouse to locate a triangular marker such that the upward facing vertex indicated the position along the VAS that would best reflect the magnitude and direction of their perception within the domain. We then recorded answers to VAS questions under each alignment condition when subjects were standing (static) and immediately after they walked along a clinic hallway for 6 to 8 steps at a self-selected speed (dynamic). Subjects were given enough time to review and adjust each VAS question until they were satisfied. They were allowed to walk additional steps if necessary.

\section{Statistical Analysis}

The PAPI measures represented a continuous response of each subject to the range of alignment perturbations induced during trials using the VAS. We first performed a Kolmogorov-Smirnov test of normality to determine the normality of the data. We then performed the Friedman test (nonparametric repeated measures analysis of variance) to investigate the statistical significance of the effect of alignment on the PAPI measures. We subsequently calculated the Dunn posttest for significance for conditions that had a $p$-value of $<0.05$. We analyzed each alignment perturbation (coronal angle and translation and sagittal angle and translation) independently with intended PAPI measures (Table 1). Statistically significant differences in this study indicate that subjects' perception of alignment has revealed significant differences between the alignment conditions.

We organized subjects' perceptions as measured by the PAPI variables into three groups: "intended," "neutral," and "unintended." Intended indicates that subjects perceived the intended effect of the alignment change (e.g., feeling of hyperextension of the knee caused by 
socket extension), while unintended indicates that they perceived the unintended (opposite) effect (e.g., feeling of walking on inside of foot despite socket abduction) (Table 2). We defined neutral responses as those where the response was within the range of \pm 2.5 in the VAS. The range of \pm 2.5 was arbitrarily given. This presumed an absence of decisive sensation in either direction. It also accounted for any marking error near the zero datum of the VAS. We analyzed the intended and unintended sensations as contingency tables using the Fisher exact test on chi-square distribution to determine the $p$-value for the observed distributions. We also calculated sensitivity and specificity of responses relative to alignment direction. We arbitrarily chose the positive and negative direction of the alignment planes so that the correct intended subject responses were either a true positive or true negative and the incorrect unintended subject responses were either a false positive or false negative (Table 2). Finally, we calculated the likelihood ratio to indicate the chance of correct perception as a multiple of the chance of incorrect perception. Table 3 shows the methods to calculate sensitivity, specificity, and likelihood ratio.

\section{RESULTS}

\section{Normality of PAPI Responses}

The Kolmogorov-Smirnov test of normality revealed that the PAPI responses would not follow a normal Gaussian distribution. Therefore, we subsequently applied a nonparametric statistical analysis (Friedman test).

\section{Effect of Alignment on PAPI Measures}

Subjects were most consistently able to detect changes with the coronal angulation. In the coronal plane, the Friedman test demonstrated general statistical differences in static $(p<0.001)$ and dynamic $(p<0.001)$ PAPI measures of perceptions with angular perturbations. The Dunn posttest did not show any significant difference between the subjects' perception of the aligned condition versus that of each induced malalignment perturbation. However, the subjects were able to differentiate how the alignment felt when comparing more extreme differences in malalignment (Figure 2). Under the static condition, the following two combinations showed significant differences: $6^{\circ}$ abduction versus $3^{\circ}$ adduction $(p<0.05)$ and $6^{\circ}$ abduction versus $6^{\circ}$ adduction $(p<0.001)$. Under the dynamic condition, the following four combinations revealed significant differences: $6^{\circ}$ abduction versus $3^{\circ}$

Table 2.

Demonstrating association between true positives and negatives and false positives and negatives with subjects' selection of direction of alignment using Prosthesis Alignment Perception Instrument (PAPI). Positive (+) and negative (-) direction for each plane was arbitrarily chosen. Subjects’ correct intended selections and incorrect unintended selections were used to calculate sensitivity, specificity, and likelihood ratio in Table 3.

\begin{tabular}{|c|c|c|}
\hline Actual Alignment Direction & Subject Selected Direction (PAPI) & Result Category \\
\hline \multicolumn{3}{|l|}{ Coronal Plane } \\
\hline Abduction + & Abduction + & Intended/True positive \\
\hline Abduction + & Adduction - & Unintended/False negative \\
\hline Adduction - & Adduction - & Intended/True negative \\
\hline Adduction - & Abduction + & Unintended/False positive \\
\hline Medial translation + & Medial translation + & Intended/True positive \\
\hline Lateral translation - & Medial translation + & Unintended/False positive \\
\hline \multicolumn{3}{|l|}{ Sagittal Plane } \\
\hline Extension + & Extension + & Intended/True positive \\
\hline Extension + & Flexion - & Unintended/False negative \\
\hline Flexion - & Flexion - & Intended/True negative \\
\hline Anterior translation - & Anterior translation - & Intended/True negative \\
\hline Anterior translation - & Posterior translation + & Unintended/False positive \\
\hline
\end{tabular}


JRRD, Volume 49, Number 6, 2012

Table 3.

Significance, sensitivity, specificity, and likelihood ratio of coronal and sagittal Prosthesis Alignment Perception Instrument (PAPI) measures with alignments examined by Fisher's exact test.

\begin{tabular}{|c|c|c|c|c|}
\hline \multirow{2}{*}{ Coronal Plane } & \multicolumn{2}{|c|}{ Coronal Angle } & \multicolumn{2}{|c|}{ Coronal Translation } \\
\hline & Coronal Static & Coronal Dynamic & Coronal Static & Coronal Dynamic \\
\hline$\overline{p \text {-Value }}$ & $<0.001^{*}$ & $<0.001^{*}$ & 0.19 & $0.02^{*}$ \\
\hline $\begin{array}{l}\text { Sensitivity (true positives/ } \\
\text { [true positives }+ \text { false negatives]) }\end{array}$ & 0.7368 (Abd) & 0.9412 (Abd) & 0.5000 (Med) & 0.8333 (Med) \\
\hline $\begin{array}{l}\text { Specificity (true negatives/ } \\
\text { [true negatives + false positives]) }\end{array}$ & 0.8500 (Add) & 0.9375 (Add) & 0.7273 (Lat) & 0.7059 (Lat) \\
\hline
\end{tabular}

[1 - specificity])

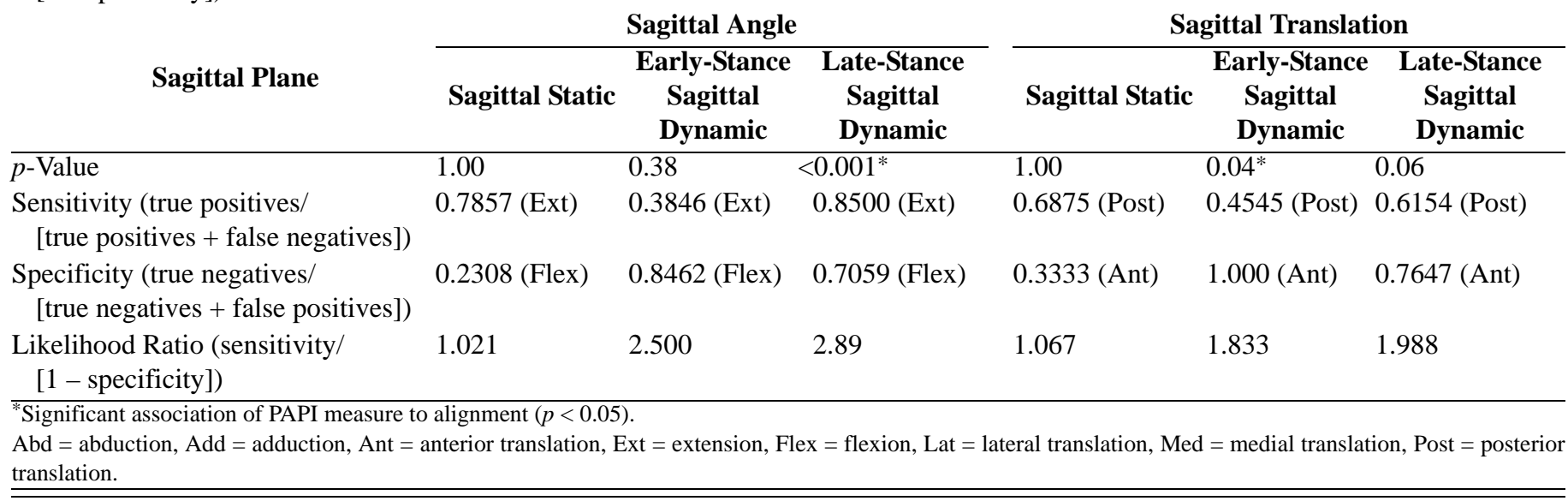

adduction $(p<0.001), 6^{\circ}$ abduction versus $6^{\circ}$ adduction $(p<0.001), 3^{\circ}$ abduction versus $3^{\circ}$ adduction $(p<0.05)$, and $3^{\circ}$ abduction versus $6^{\circ}$ adduction $(p<0.01)$. The coronal translation was the most inconsistent testing condition with regard to subjects' perceptions of the alignment. The Friedman test did not demonstrate general statistical differences in the static and dynamic PAPI measures with translational perturbations in the coronal plane.

In the sagittal plane, the Friedman test also demonstrated general statistical differences in late-stance dynamic PAPI measures of perceptions $(p<0.001)$ with angular perturbations, as well as in early-stance dynamic PAPI measures of perceptions $(p<0.05)$ with translational perturbations. Again, the Dunn posttest indicates that this is caused by the extremes of malalignment rather than smaller perturbations from the aligned condition (Figure 2). The following two combinations showed significant differences: $3^{\circ}$ extension versus $6^{\circ}$ flexion $(p<$ $0.01)$ and $6^{\circ}$ extension versus $6^{\circ}$ flexion $(p<0.01)$. The PAPI early-stance sagittal dynamic measure varied significantly with sagittal translation $(p<0.05)$. Only one combination revealed significant differences: $10 \mathrm{~mm}$ posterior translation versus $10 \mathrm{~mm}$ anterior translation $(p<0.05)$. Figure 2 shows the quartile distribution of the PAPI measures that were found to vary significantly ( $p<$ 0.05 ) with changes in alignment (coronal static, coronal dynamic, late-stance sagittal dynamic, and early-stance sagittal dynamic) by the Friedman test.

\section{Association of PAPI Measures to Malalignments}

The Fisher exact test revealed significant association of the coronal dynamic PAPI measures to the coronal angular $(p<0.001)$ and coronal translational $(p<0.05)$ perturbations as well as the coronal static PAPI measures to coronal angle perturbations $(p<0.001)$. The Fisher exact test also showed significant association of the latestance sagittal dynamic PAPI measures to the sagittal anglular $(p<0.001)$ perturbations as well as the earlystance sagittal dynamic to the sagittal translational $(p<$ 0.05 ) perturbations. Figure 3 plots histograms of the frequency distributions of the PAPI measures that demonstrated a significant association with alignment. The horizontal axis of each graph in Figure $\mathbf{3}$ is an actual alignment perturbation induced, while the vertical axis is the number of intended or unintended responses. 

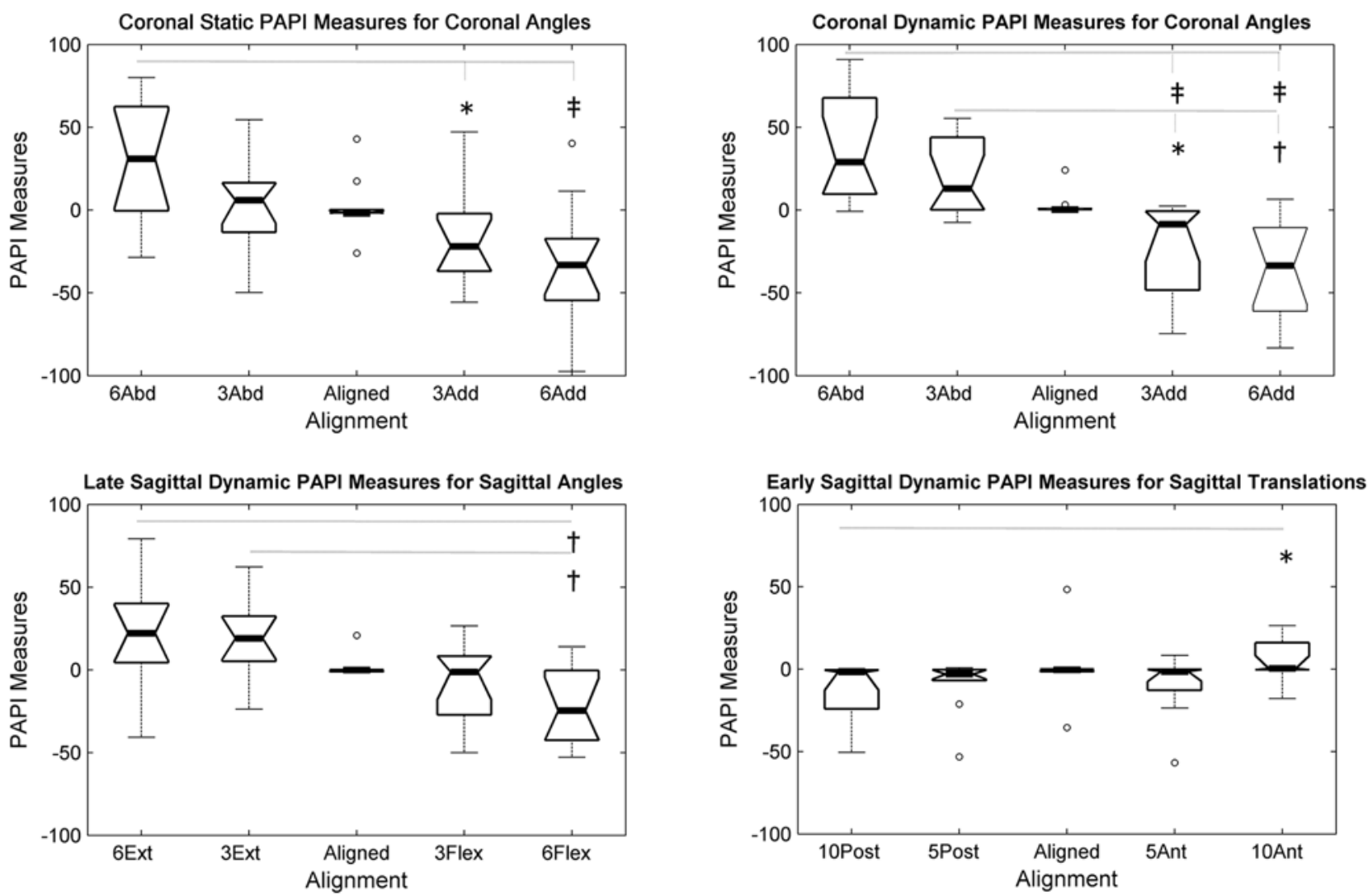

Figure 2.

Quartile distribution of Prosthetics Alignment Perception Instrument (PAPI) measures that were found to vary significantly $(p<0.05)$ by Friedman's test with changes in alignment (coronal static, coronal dynamic, late-stance sagittal dynamic, and early-stance sagittal dynamic). Boxes represent central 50 percent of distribution. Vertical whiskers extend to values up to 1.5 interquartile range. Outliers are plotted as open circle. ${ }^{*}$ Indicates significance level of $p<0.05$. ${ }^{\dagger}$ Indicates significance level of $p<0.01$. ${ }^{\ddagger}$ Indicates significance level of $p<0.001$. Abd $=$ abduction, Add $=$ adduction, Ant $=$ anterior translation, Ext $=$ extension, Flex $=$ flexion, Lat $=$ lateral translation, Med $=$ medial translation, Post $=$ posterior translation

\section{Sensitivity, Specificity, and Likelihood Ratio of PAPI Measures with Malalignments}

Table 3 summarizes sensitivity, specificity, and likelihood ratio outcomes of the coronal PAPI measures. The Fisher exact test revealed that the malalignment perceptions of subjects were very good as an indicator of the general coronal angulation malalignment with a likelihood ratio of 15.1 for the dynamic coronal measure and 4.9 for the static coronal measure. Therefore, the likelihood of a correct perception by the subject was demonstrated to be 15 times the likelihood of an incorrect one. The likelihood ratio of the dynamic coronal PAPI measures was 2.8 for coronal translations and that of the late-stance sagittal dynamic PAPI measures was 2.9 for sagittal angulations.
The likelihood ratio of the early-stance sagittal dynamic PAPI to sagittal translations was 1.8. The ratio of the intended (true positives or true negatives), neutral, and unintended (false positives or false negatives) can be graphically viewed in Figure 3.

\section{DISCUSSION}

The aim of this study was to investigate whether individuals with transtibial prostheses could perceive the alignment perturbations of their prostheses and communicate them effectively. In general, there were statistically significant measures that reflected subjects' ability to 
JRRD, Volume 49, Number 6, 2012
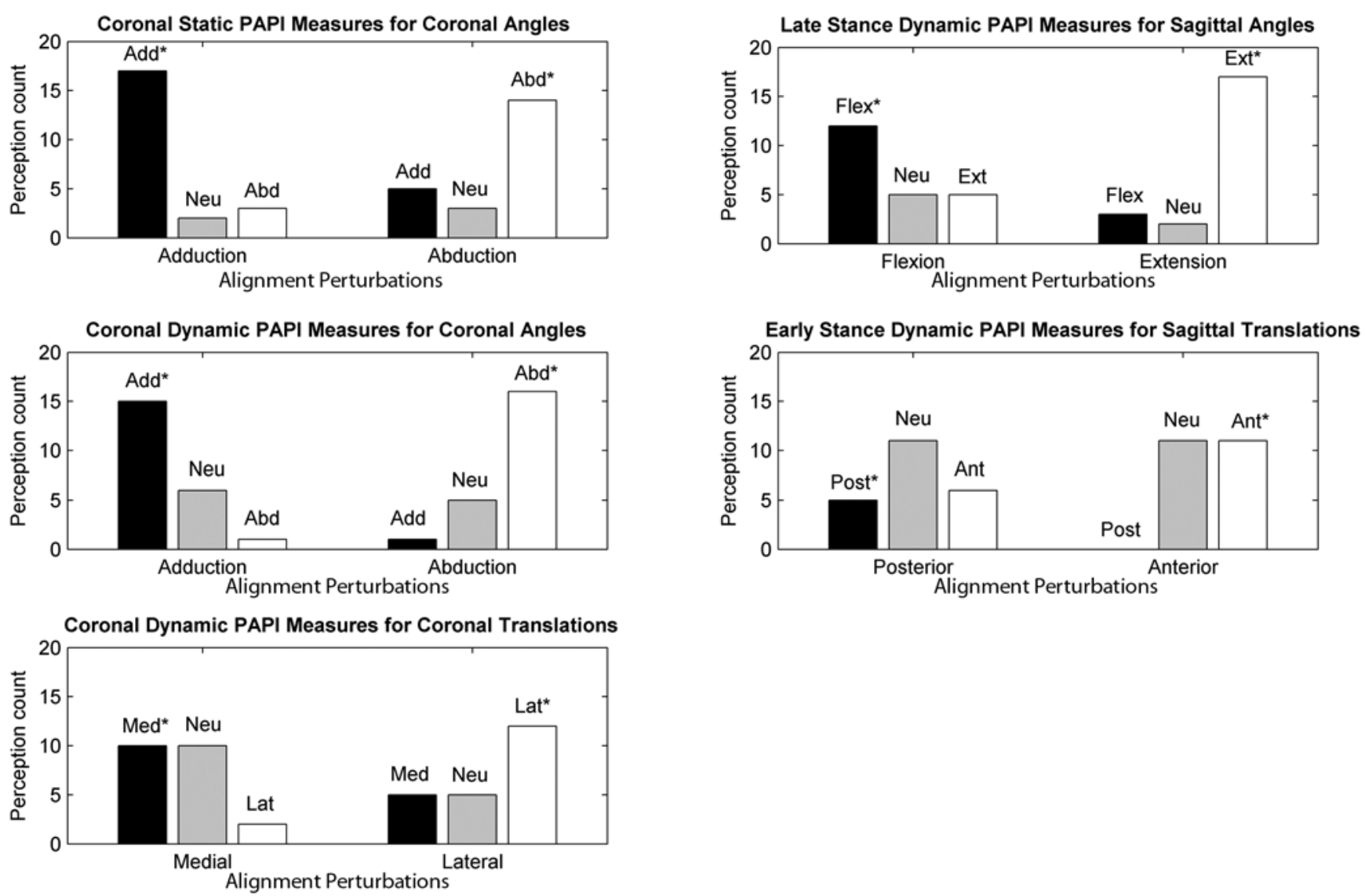

Figure 3.

Histograms of frequency distributions of Prosthetics Alignment Perception Instrument (PAPI) measures that demonstrated significant association with alignment. Horizontal axis of each graph is actual alignment perturbations, while vertical axis is number of intended, neutral, and unintended responses. ${ }^{*}$ Indicates intended (true positives or true negatives) response. Abd = abduction, Add = adduction, Ant $=$ anterior translation, Ext $=$ extension, Flex $=$ flexion, Lat $=$ lateral translation, Med $=$ medial translation, Neu $=$ neutral, Post $=$ pos terior translation.

correctly communicate the more extreme differences between alignment conditions. Measurement of subjects' perceptions of the prosthetic alignment also complemented our previous work that demonstrated the systematic effect of alignment perturbations on socket reaction moments in the sagittal and coronal planes [22].

The PAPI measures worked better for dynamic perceptions than static perceptions. The subjects were easily able to maintain balance statically despite malalignment. They might have attained an artificial sense of the alignment using an accommodative strategy, such as repositioning of the prosthetic foot and redistribution of the bodyweight to the contralateral side. The foot position and weight distribution between feet were not controlled, and it would obviously be more difficult for a person with amputation to perform a similar accommodative strategy dynamically.

Another factor that would significantly affect loading strategies would be the distribution of hard and soft tissue as a result of the original surgical approach at the time of amputation as well as any subsequent revisions. Variations in compliant versus hard tissue will result in distinctive sensations and areas of sensitivity related to socket interface stress distributions. However, it is still unknown whether a more lean or fleshy residual limb may provide better sensatory capacity to the person with amputation.

Perception of prosthetic alignment revealed the strongest agreement with the coronal angulations. Angular changes may have greater effect of linear placement of the foot than some translational changes based on the 
limb length and distance from the socket adaptor to the floor. However, the Dunn posttest did not show any significant difference between the aligned conditions and induced perturbations. This indicated that malalignments of less than $6^{\circ}$ might be "subclinical" in a person with amputation's perception. The coronal static PAPI measures revealed a significant difference between $6^{\circ}$ abduction and $3^{\circ}$ adduction and between $6^{\circ}$ abduction and $6^{\circ}$ adduction (Figure 2), while the coronal dynamic PAPI measures revealed a significant difference between $3^{\circ}$ abduction and $3^{\circ}$ adduction, between $6^{\circ}$ abduction and $3^{\circ}$ adduction, between $3^{\circ}$ abduction and $6^{\circ}$ adduction, and between $6^{\circ}$ abduction and $6^{\circ}$ adduction (Figure 2). Although not all subjects perceived the malalignment precisely, the important finding here was that if a person with amputation perceived a malalignment leading to socket abduction or adduction, then the malalignment would exist in the direction he or she perceived.

The results of this study also suggest that the perception of the coronal translation is much weaker than that of the coronal angulation. This result also agrees with a preceding study demonstrating that coronal angulation would induce more socket interface pressure changes than coronal translations [21]. This is an interesting finding considering that the socket reaction moments varied similarly between the coronal angle and translation conditions as measured with the instrumented prosthesis alignment component [22]. One would expect a comparable dynamic perception outcome. This may be explained by the fact that it is comparatively easy for a person with amputation to use hip rotation $\left(\approx 0.6^{\circ}\right)$ to translate the foot $10 \mathrm{~mm}$ in order to offset the maximal translation perturbation made. However, the maximum angular perturbation of $6^{\circ}$ is an order of magnitude greater. Therefore, an accommodation that is 10 times greater would be demanded for him or her to keep the foot flat on the floor.

The sagittal translation and angulation malalignments were perceived as having effects at opposite ends of the stance phase, with the translation effects being felt in early dynamic stance and angular effects being perceived in late dynamic stance (Figure 3). The late-stance sagittal dynamic PAPI measures revealed a significant difference between $3^{\circ}$ extension and $6^{\circ}$ flexion and between $6^{\circ}$ extension and $6^{\circ}$ flexion, while the earlystance sagittal dynamic PAPI measures revealed a significant difference between $10 \mathrm{~mm}$ posterior and $10 \mathrm{~mm}$ anterior translations (Figure 2).
Noting the decrease in variance of the PAPI measures around the nominally aligned condition is also important. The quartile distribution demonstrated a clear trend for a more consistent response pattern near the aligned condition (Figure 2). While some subjects might have responded with neutral responses when there actually was an alignment perturbation, all subjects had nearly neutral perceptions of the aligned case.

The study is not without limitations. First, this study was specific to subjects with transtibial prostheses. The perception was only measured during stance phase, while it should also be measured during swing phase for those with transfemoral prostheses. Second, the nominally aligned condition was determined by the prosthetist and the subject. However, this might not have been the most "optimal" alignment as "true optimal” is not universally defined. Third, only one female subject participated in this study. It is not known whether there is any sex difference for the perception of the malalignment of a prosthesis. Fourth, the etiology of the subject population, 91 percent trauma, is not representative of the transtibial population as a whole. A future study should investigate (1) what type of feedback might be valuable and what type might be misleading when tuning an alignment, (2) the prosthetist's perception of different malalignments, (3) the effect of alignment perturbations on subjects with transfemoral amputation, (4) sex differences with regard to sensitivity to alignment perturbations, and (5) the effect of etiology of amputation on perception of alignment.

The results supported the stated hypothesis. We could reject the null hypothesis that individuals with transtibial prostheses would not perceive prosthetic malalignment and would not be able to communicate the nature of the malalignment in the coronal and sagittal planes using the PAPI. If a subject perceived a malalignment, then it was most likely that the malalignment would exist in the direction perceived. However, the subject's perception measured by the PAPI was not accurate enough to detect small ( $<6^{\circ}$ angulation, $<20 \mathrm{~mm}$ translation) yet potentially clinically important malalignments.

\section{CONCLUSIONS}

This study measured the variability in the perception of prosthetic malalignment. The Friedman test demonstrated that the subjects' perceptions of the alignment would reveal a general statistical difference among the 
more extreme malalignment conditions induced by angular or translational perturbations in the coronal and sagittal planes. The Fisher exact test demonstrated that subjects' perceptions of the malalignment might serve as a good indicator for the coronal angular malalignment with likelihood ratios of 4.9 (static condition) and 15.1 (dynamic condition). However, it might only be somewhat helpful for other types of alignment. The relatively weak sensitivity and specificity of the subjects to detect sagittal malalignment when changes in the socket reaction moments showed statistical significant differences [22] would indicate an example where instrumentation would be beneficial to objectively establish an optimum alignment.

\section{ACKNOWLEDGMENTS}

\author{
Author Contributions: \\ Study concept and design: D. A. Boone, M. Zhang. \\ Acquisition of data: D. A. Boone, T. Kobayashi, M. Zhang. \\ Analysis and interpretation of data: D. A. Boone, T. Kobayashi, \\ T. G. Chou, A. K. Arabian, M. Zhang, K. L. Coleman, M. S. Orendurff. \\ Drafting of manuscript: D. A. Boone, T. Kobayashi, T. G. Chou, \\ A. K. Arabian, K. L. Coleman, M. S. Orendurff. \\ Critical revision of manuscript for important intellectual content: \\ D. A. Boone, T. Kobayashi, T. G. Chou, A. K. Arabian, M. Zhang, \\ K. L. Coleman, M. S. Orendurff. \\ Obtained funding: D. A. Boone, M. Zhang. \\ Study supervision: M. Zhang.
}

Financial Contributions: Drs. Boone, Kobayashi, Chou, Arabian, and Orendurff are currently employees of the company that manufactures the Smart Pyramid, a system to assist the adjustment of alignment for a lower-limb prosthesis. However, there is no commercial interest in or from this research. The supporting sources (The Hong Kong Polytechnic University and Orthocare Innovations) had no influence on the study design or analysis.

Funding/Support: This material was based on work supported by an internal research grant of The Hong Kong Polytechnic University.

Additional Contributions: Drs. Boone and Kobayashi are no longer with The Hong Kong Polytechnic University.

Institutional Review: This study was approved by the Human Subjects Ethics Committee of The Hong Kong Polytechnic University, and informed consent was obtained from each subject.

Participant Follow-Up: The authors do not plan to inform participants of the publication of this study because contact information is unavailable.

\section{REFERENCES}

1. Hannah RE, Morrison JB, Chapman AE. Prostheses alignment: effect on gait of persons with below-knee amputa- tions. Arch Phys Med Rehabil. 1984;65(4):159-62. [PMID:6712431]

2. Zahedi MS, Spence WD, Solomonidis SE, Paul JP. Alignment of lower-limb prostheses. J Rehabil Res Dev. 1986; 23(2):2-19. [PMID:3723422]

3. James WV. Principles of limb fitting and prostheses. Ann R Coll Surg Engl. 1991;73(3):158-62. [PMID:2042893]

4. Klute GK, Kantor C, Darrouzet C, Wild H, Wilkinson S, Iveljic S, Creasey G. Lower-limb amputee needs assessment using multistakeholder focus-group approach. J Rehabil Res Dev. 2009;46(3):293-304. [PMID:19675983]

http://dx.doi.org/10.1682/JRRD.2008.02.0031

5. Pinzur MS, Cox W, Kaiser J, Morris T, Patwardhan A, Vrbos L. The effect of prosthetic alignment on relative limb loading in persons with trans-tibial amputation: a preliminary report. J Rehabil Res Dev. 1995;32(4):373-77. [PMID:8770802]

6. Fernie GF. Biomechanics of gait and prosthetic alignment. In: Kostuik JP, Gillespie R, editors. Amputation surgery and rehabilitation: The Toronto experience. New York (NY): Churchill Livingstone; 1981. p. 259-65.

7. Hanak R, Berger N. Lower limb prosthetics prosthetist's supplement. New York (NY): New York University, PostGraduate Medical School; 1990. p. 119.

8. Cummings D, Kapp S, McClellan B. Below knee prosthetics: Prosthetics-orthotics program. Dallas (TX): University of Texas Southern Medical Center at Dallas; 1998.

9. Buckley JG, Jones SF, Birch KM. Oxygen consumption during ambulation: comparison of using a prosthesis fitted with and without a tele-torsion device. Arch Phys Med Rehabil. 2002;83(4):576-80. [PMID:11932866] http://dx.doi.org/10.1053/apmr.2002.30624

10. Coleman KL, Boone DA, Laing LS, Mathews DE, Smith DG. Quantification of prosthetic outcomes: elastomeric gel liner with locking pin suspension versus polyethylene foam liner with neoprene sleeve suspension. J Rehabil Res Dev. 2004;41(4):591-602. [PMID:15558387] http://dx.doi.org/10.1682/JRRD.2004.04.0591

11. Datta D, Howitt J. Conventional versus microchip controlled pneumatic swing phase control for trans-femoral amputees: user's verdict. Prosthet Orthot Int. 1998;22(2): 129-35. [PMID:9747997]

12. Kaufman KR, Levine JA, Brey RH, McCrady SK, Padgett DJ, Joyner MJ. Energy expenditure and activity of transfemoral amputees using mechanical and microprocessorcontrolled prosthetic knees. Arch Phys Med Rehabil. 2008; 89(7):1380-85. [PMID:18586142] http://dx.doi.org/10.1016/j.apmr.2007.11.053

13. Bateni H, Olney SJ. Effect of the weight of prosthetic component on the gait of transtibial amputees. J Prosthet Orthot. 2004;16(4):113-20. http://dx.doi.org/10.1097/00008526-200410000-00004 
14. Macfarlane PA, Nielsen DH, Shurr DG, Meier K. Perception of walking difficulty by below-knee amputees using a conventional foot versus the flex-foot. J Prosthet Orthot. 1991;3(3):114-19. http://dx.doi.org/10.1097/00008526-199106000-00007

15. Underwood HA, Tokuno CD, Eng JJ. A comparison of two prosthetic feet on the multi-joint and multi-plane kinetic gait compensations in individuals with a unilateral transtibial amputation. Clin Biomech (Bristol, Avon). 2004; 19(6):609-16. [PMID:15234485]

http://dx.doi.org/10.1016/j.clinbiomech.2004.02.005

16. Fradet L, Alimusaj M, Braatz F, Wolf SI. Biomechanical analysis of ramp ambulation of transtibial amputees with an adaptive ankle foot system. Gait Posture. 2010;32(2): 191-98. [PMID:20457526] http://dx.doi.org/10.1016/j.gaitpost.2010.04.011

17. Boone DA, Coleman KL. Use of the prosthesis evaluation questionnaire (PEQ). J Prosthet Orthot. 2006;18(6):P68-P79. http://dx.doi.org/10.1097/00008526-200601001-00008

18. Hafner BJ, Sanders JE, Czerniecki J, Fergason J. Energy storage and return prostheses: does patient perception correlate with biomechanical analysis? Clin Biomech (Bristol, Avon). 2002;17(5):325-44. [PMID:12084537] http://dx.doi.org/10.1016/S0268-0033(02)00020-7

19. Hobson D. A powered aid for aligning the lower-limb modular prosthesis. Bull Prosthet Res. 1972;10(18):159-63.

20. Burnfield J, Bontrager E, Boyd L, Rao S, Mulroy S, Gronely J, Perry J. Effect of prosthetic malalignment on
EMG and intrasocket pressures in an individual with a trans-tibial amputation. Gait Posture. 1999;9(2):137-38.

21. Zhang M, Turner-Smith AR, Tanner A, Roberts VC. Clinical investigation of the pressure and shear stress on the trans-tibial stump with a prosthesis. Med Eng Phys. 1998; 20(3):188-98. [PMID:9690489] http://dx.doi.org/10.1016/S1350-4533(98)00013-7

22. Boone DA. Investigation of socket reactions from transtibial prosthesis malalignment [thesis]. [Hong Kong]: The Hong Kong Polytechnic University; 2005. 272 p. Available at: http://repository.lib.polyu.edu.hk/jspui/handle/10397/3903

Submitted for publication August 12, 2011. Accepted in revised form November 16, 2011.

This article and any supplementary material should be cited as follows:

Boone DA, Kobayashi T, Chou TG, Arabian AK, Coleman KL, Orendurff MS, Zhang M. Perception of socket alignment perturbations in amputees with transtibial prostheses. J Rehabil Res Dev. 2012;49(6):843-54.

http://dx.doi.org/10.1682/JRRD.2011.08.0143

Researcher ID: David A. Boone, PhD: E-2255-2012

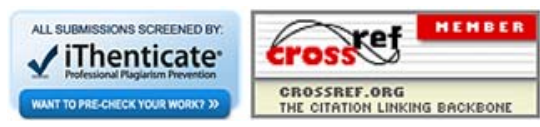


